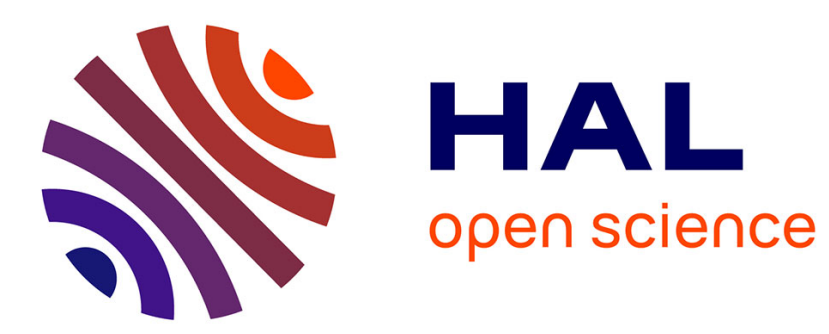

\title{
A modified Panjer algorithm for operational risk capital calculations
}

Dominique Guegan, Bertrand Hassani

\section{To cite this version:}

Dominique Guegan, Bertrand Hassani. A modified Panjer algorithm for operational risk capital calculations. Journal of Operational Risk, 2009, 4 (4), pp.53-72. halshs-00443846

\section{HAL Id: halshs-00443846 \\ https://shs.hal.science/halshs-00443846}

Submitted on 5 Jan 2010

HAL is a multi-disciplinary open access archive for the deposit and dissemination of scientific research documents, whether they are published or not. The documents may come from teaching and research institutions in France or abroad, or from public or private research centers.
L'archive ouverte pluridisciplinaire HAL, est destinée au dépôt et à la diffusion de documents scientifiques de niveau recherche, publiés ou non, émanant des établissements d'enseignement et de recherche français ou étrangers, des laboratoires publics ou privés. 


\section{A modified Panjer algorithm for operational risk capital calculations}

November 5, 2009

\section{Authors}

- D. Guégan: Paris School of Economics, CES-MSE, Université Paris 1 PanthéonSorbonne, 106 boulevard de l'Hopital 75647 Paris Cedex 13, France, e-mail: dguegan@univ-paris1.fr

- B. Hassani1: BPCE and CES-MSE, Université Paris 1 Panthéon-Sorbonne, 106 boulevard de l'Hopital 75647 Paris Cedex 13, phone: +33 (0) 158403397 France, email: bertrand.hassani@bpce.fr 


\section{Executive Summary}

Operational risk management inside banks and insurance companies is currently an important task. The computation of a risk measure associated to these kinds of risks lies on the knowledge of the so-called Loss Distribution Function (LDF). Traditionally this LDF is computed via Monte Carlo simulations or using the Panjer recursion which is an iterative algorithm. In this paper, we propose an adaptation of this last algorithm in order to improve the computation of convolutions between Panjer class distributions and continuous distributions, by mixing the Monte Carlo method, a progressive kernel lattice and the Panjer recursion. This new hybrid algorithm does not face the traditional drawbacks. This simple approach enables us to drastically reduce the variance of the estimated VaR associated to the operational risks and, to lower the aliasing error we would have using Panjer recursion itself. Furthermore, this method is much less time-consuming than a Monte Carlo simulation. We compare our new method with more sophisticated approaches already developed in operational risk literature.

Keywords: Operational risk - Panjer algorithm - Kernel - Numerical integration - Convolution. 


\section{Introduction}

The purpose of this article is - among the Basel Advanced Measurement Approach framework to assess a Value-at-Risk measure from historical data on operational risk. Indeed, the Basel II accords propose financial institutions to develop internal models in order to evaluate operational risks exposition and a fortiori manage them. Our purpose is to contribute to this last objective proposing a novel approach to compute VaR measures.

Operational risk is a very wide concept of risk arising from the failure of a business function. It is probably the most pernicious form of risks because it is indirectly responsible of numerous failures in financial institutions. Most financial fiascos can be traced to a combination of market or credit risks and failures of controls - in other words, they involve forms of operational risks. An illustration of such a risk is rogue trading, for example the spectacular bankrups of the Barings bank and others involving rogue trading can be attributed to operational risk. Another one is internal frauds which arise at the highest level of the organization and are due to poor corporate governance. It seems useful to recall extremely large operational risk losses: Barings Bank in 1995, Sumitomo Corporation in 1996, Enron in 2001, Société Générale in 2008 and Group Caisse d'Epargne in 2008. These huge losses inside banks have increased the interest for operational risk management.

The computation of operational risks depends on the so-called distribution function [Frachot et al. (2001)]. The literature proposes several methods to compute it. The loss distribution function that enables us to characterize the severities and the frequencies of the specific events, has no closed-form expression because it is generally calculated as a compound distribution. Two kinds of distributions are mainly used to model the events that are associated to operational risks: the discrete distribution functions such as Poisson, Binomial or Negative Binomial distributions for modelling the frequency of events and the continuous distribution functions - for instance Lognormal, Weibull, Gumbel, Generalized Pareto (GPD) or g-and-h distributions - to characterize the severity of these events ([Pickands (1975)], [Hoaglin (1985)], [Buch-Kroman (2009)]). To compute such compound distributions, convolution methods associated with Monte Carlo simulations are extensively used. Traditional "exact" convolution methods are based on the Fast 
Fourier Transform [Cooley and Tukey (1965)], or on the Heckman-Myers algorithm through the Laplace transform [Heckman and Meyers (1983)] or finally on the Panjer's algorithm ([Panjer (1981)] and [Embrechts and Frei (2009)]). Recently, to bypass some limits of these methods, [Luo and Shevchenko (2009)] and [Peters et al. (2007)] developped new algorithms to proceed in VaR estimation. We develop in this paper an aternative approach which reduce errors inherent to the algorithms developed in the previous cited works.

Our approach mixes the use of the Panjer algorithm [Panjer (1981)] with kernel density estimation [Wand and Jones (1995)], and Monte Carlo simulations [Fishman (1996)]. It improves the accuracy of the VaR estimation and makes the computations faster than with classical methods. With this approach, we avoid problems due to the high frequencies of the loss, mainly computing's limit or lack of convergence of algorithms. Another important task in this paper concerns the variance reduction of the loss distribution. The computation speed of Panjer algorithm is interesting, but in all cases it remains an initialization problem. We solve this problem increasing the size of the information set avoiding the lack of robustness encountered in most classical methods. To do so, we build a partial lattice which is our first information set using Monte Carlo simulations using nonparametric kernel smoothing and then we apply the Panjer algorithm, avoiding evaluating initial integrals with a too small number of intermediary points.

The paper is organized as follows. In Section 1 we recall the notion of operational risk, Loss Distribution Function and risk measure. Section 2 presents alternative methods to compute the VaR. Section 3 presents the methodology we use to approximate the Loss Distribution Function and specifies the numerical integration error. Section 4 presents experiments which illustrate the interest of our methodology. Section 5 concludes.

\section{Definitions}

\subsection{Framework}

An operational risk is a risk arising from execution of company's business functions, as such, it is a very wide concept including risks such as legal, physical or environmental frauds. The notion of operational risk is commonly found in risk management programs of financial institutions. 
In many cases, credit and market risks are handled through a company's financial department, whereas operational risk management is coordinated centrally but most of the time divided and located in different operational units which makes difficult the control of this kind of risks.

The Basel Committee specifies the operational risk as: "The risk of loss resulting from inadequate or failed internal processes, people and systems or from external events." This way of framing risk management is of particular relevance for the banking regime where regulators are responsible for establishing safeguards to protect institutions against the systemic failure of the banking system and the economy. The Basel II definition of operational risk clearly excludes strategic risk and reputation risk.

Basel accords demand to use different ways to quantify operational risk. We can distinguish the scenarios which enable us to access losses due to extremal risks and the use of historical data (internal and external source) to compute a Loss Distribution Function. Here we focus on the computation of the LDF considering it as a compound of the loss severity distribution function $F_{b, e}$, (" $b "$ corresponding to the business line and " $e$ " to the event type), and of the loss frequency distribution function $p_{b, e}$. Our approach provides a statistical and numerical solution computing it, and we use it to compute the VaR associated to the distribution function [Morgan (1993)].

\subsection{The loss distribution function (LDF)}

As a matter of fact, loss is most of the time positive. Some specific insurance contracts might reverse the fact and we could face gains, but it is very unsual. For stability reasons, we will assume that we have only losses. Therefore, the major part of those are concentrated on the right tail of the distribution function.

On our severities data (Section 3), we have used goodness-of-fit tests such as KolmogorovSmirnov, Cramer Von Mises and Anderson-Darling at level of $95 \%$ to detect the "best" distributions which characterize these data sets. We have retained a Lognormal distribution. Nevertheless to give the opportunity to practitioners to use our approach with other distributions, we consider in the following the Weibull one and the GPD one to model the severities (Embrechts 
et al. (2007)). In order to achieve the Panjer recursion scheme which is fundamental in our approach, following the previous cited works, we focus on distributions satifying the recursion $p_{n}=p_{n-1}\left(a+\frac{b}{n}\right)$, i.e. Poisson, Binomial, Negative Binomial or Geometric distribution. In our examples, we consider only Poisson and Binomial distributions to characterize the frequencies. We introduce now the densities and mass functions of the distributions we use to model the LDF:

- LogNormal distribution: $f_{b, e}(x ; \mu, \sigma)=\frac{1}{x \sigma \sqrt{2 \pi}} e^{-\frac{(\ln (x)-\mu)^{2}}{2 \sigma^{2}}}$ for $x>0, \mu \in \mathrm{R}, \sigma>0$.

- Weibull distribution: $f_{b, e}(x ; \alpha, \theta)=\frac{\alpha}{\theta} \frac{x}{\theta}{ }^{\alpha-1} e^{-\left(\frac{x}{\theta}\right)^{\alpha}}$ for $x>0, \alpha>0, \theta>0$.

- Generalized Pareto distribution: $f_{b, e}(x, \eta, \beta, \alpha)=\frac{1}{\beta}\left(1+\alpha \frac{(x-\eta)}{\beta}\right)^{\left(-1-\frac{1}{\alpha}\right)}$ with $1+\alpha \frac{(x-\eta)}{\beta}>$ $0, \beta>0$ and $\eta \neq 0$ (or $f_{b, e}(x, \eta, \beta, \alpha)=\frac{1}{\beta}\left(-\frac{(x-\eta)}{\beta}\right)$ if $\left.\alpha=0\right)$.

- Poisson distribution: $p_{b, e}(k ; \lambda)=e^{-\lambda} \frac{\lambda^{k}}{k !}$ where $\lambda \in \mathrm{R}^{+}$and $k \in \mathrm{N}$.

- Binomial distribution: $p_{b, e}(k ; n, p)=\left(\begin{array}{c}n \\ k\end{array}\right) p^{x}(1-p)^{(n-k)}$ where $p \in[0,1]$ and $k \in \mathrm{N}$.

Finally, the loss distribution function $G_{b, e}$, which appears as the mixture of two of the previous distribution functions is equal to:

$$
G_{b, e}=\sum_{k=1}^{\infty} p_{b, e}(k, \lambda) F_{b, e}^{\otimes k}, x>0,
$$

with

$$
G_{b, e}=0, x=0
$$

Here $\otimes$ denotes the convolution operator between distribution functions and $F_{b, e}^{\otimes n}$ the $n$-fold convolution of $F_{b, e}$ with itself. We denote $g_{b, e}$ the density function associated to $G_{b, e}$, then:

$$
g_{b, e}=\sum_{k=1}^{\infty} p_{b, e}(k, \lambda) f_{b, e}^{\otimes k}, x>0 .
$$

In practice, we will consider a discretization of (1.2) in the algorithm we develop in Section 3.

\subsection{Value-at-Risk measure}

In financial mathematics and financial risk management, Value-at-Risk (VaR) measure is popular and we use it here, even if it is known as a non-coherent measure [Artzner et al. (1999)]. Formally the VaR is defined as follows: 
Definition 1.1. Given a confidence level $\alpha \in[0,1]$, the VaR associated to a random variable $L$ is given by the smallest number $l$ such that the probability that $L$ exceeds $l$ is not larger than $(1-\alpha)$.

$$
\operatorname{VaR}_{(1-\alpha) \%}=\inf (l \in \mathcal{R}: P(L>l) \leq(1-\alpha))
$$

The regulator imposes a security threshold of $99.9 \%$, therefore $\alpha=0.1 \%$, thus, in this paper, we follow the rule.

\section{Several methods to compute the VaR}

The objective of our paper is to compute a Value-at-Risk associated to the LDF which caracterizes the losses observed in a company. Different methods have already been proposed in the litterature to solve this expectation. We recall and compare them with our methodology.

\subsection{Monte Carlo Simulations (MC)}

MC simulations [Fishman (1996)] are often used to compute the LDF. This method is famous and commonly used, even if it is a slow method. We use it in this paper, as a benchmark.

\subsection{Fast Fourier Transform approach (FFT)}

The FFT method [Schatzman (1991)] is sometimes presented as a traditional alternative to compute the LDF. Applying the FFT method requires to build an initial grid of the LDF sufficiently large to contain the VaR but not too large to avoid significant computation time. Another limit of the FFT method is the aliasing error observed when we evaluate compound losses ([Embrechts and Frei (2009)], [Schaller and Temnov (2008)] and [Grubel and Hermesmeier (1999)]). In the previous cited papers, authors applied the tilting or exponential windowing procedure to reduce this error. Even if a tilting procedure can reduce aliasing errors, in case of heavy tails and high frequencies, this method is not sufficient to reduce the discretization error. We will not consider this approach. 


\subsection{Panjer Recursion method (PR)}

The Panjer recursion method has often been compared with the FFT method, and it is accepted that the former is less time consuming as soon as the grid size is large ([Bühlmann (1984)], [Grubel and Hermesmeier (1999)], [Embrechts and Frei (2009)]). In a recent paper, [Peters et al. (2007)] use Panjer recursion noticing that the Panjer recursion can be characterized as a second type Volterra equation [Panjer (2006)] with interesting underlying properties, importance sampling and trans-dimensional Markov chain Monte Carlo to improve the efficiency of the VaR estimation. This approach is very competitive compared with our approach but suffers from too much theoretical complexity. Their algorithm is very complex and difficult to implement. In our approach, we introduce the Panjer recursion method in a different way.

\subsection{Characteristic Function approach (CF)}

The last few decades were a prosperous period in the area of numerical inversion of CF ([Heckman and Meyers (1983)], [Bühlmann (1984)], [Den Iseger (2006)], etc.). The various issues of these last papers concern mainly the treatment of long tails in infinite integration or the choice of quadrature rules. The algorithm based on the characteristic function have strengths and weakness as recalled in [Craddock et al. (2000)] and [Luo and Shevchenko (2009)]. The main drawback of the method concerns the precision of the algorithm and the numerical inversion which is time consuming. In their paper, [Luo and Shevchenko (2009)] calculate high quantile and corresponding conditional Value at Risk (CVaR) for compound distributions. Their algorithm appears efficient for highevents frequency and heavy tailed severity distributions. They consider truncation errors and use uniform grids in the numerical integration. We do not develop this approach in the current paper.

\subsection{Our approach}

In the following, we develop an algorithm which mixes Monte Carlo simulations, Panjer algorithm and kernel smoothing. We compare our results with Monte Carlo simulation (Section 2.1). Concerning the Panjer recursion method (Section 2.3), in order to avoid the non-convergence of the method due to the lack of points inside the quadrature formula, we introduce a complemen- 
tary grid that we describe in the next section.

We do not follow the method proposed by Luo and Shevchenko (2009), because their method computes only a quantile and does not provide the full distribution function which is the objective of our paper, nevertheless we compare our results of VaR value with theirs.

\section{A new numerical algorithm to compute the LDF}

In this section, we introduce a new method to compute the LDF $G_{b, e}$. As we have no closed-form for the LDF introduced in (1.1), we developed a fast, robust and precise algorithm to compute it. We introduce several new steps before applying the Panjer recursion, building recursive sequences of non-parametric densities to increase the speed and the accuracy of the algorithm. In the following, to simplify the notations, we denote $G$ the loss distribution and $g$ its density.

\subsection{Details of the procedure}

1. The first step consists in using the Monte Carlo method ([Fishman (1996)], [Chernobai et al. (2007)]):

(a) We simulate $N$ realisations for the frequency distributions.

(b) We draw i.i.d. realisations for the severity distribution $\left(L_{1}, \ldots, L_{N}\right)$.

(c) We compute $T L_{i}=\sum_{j=1}^{N} L_{j}$, a realisation of the loss distribution, $i=1, \ldots, k(\mathrm{k}$ corresponds to the order of convolution).

The set $\left(T L_{1}, \ldots, T L_{k}\right)$, provides an empirical representation of the LDF that we denote $\hat{G}$, and $\hat{g}$ its density. This first approximation of the true LDF provides a good estimation of the associated VaR measure if we consider a very large number of simulations, otherwise the variance and the error associated to the VaR will be too large. The Panjer recursion method is used to compute iteratively the convolution (1.1). The iteration is done point by point, and finally we obtain a new approximation of $G$ still denoted $\hat{G}$. But the initialization of the Panjer algorithm induces a bias in the VaR results. In order to make the method more robust, we propose now new steps increasing the information set on which the iterations are applied. This allows us to reduce the variance. 
2. Using the support of the distribution $\hat{g}$ obtained in the previous step, we construct a sequence of rolling subsets $A_{k}, k=1,2, \cdots$, on which we build non-parametric densities $\tilde{g}^{\alpha, \beta}(k)$ using Epanechnikov kernel, [Wand and Jones (1995)]. Thus, we get a sequence of densities $\left(\tilde{g}^{\alpha, \beta}(k)\right)$. Here, $\alpha$ denote the Panjer point and $\beta$ the point corresponding to the kernel grid between two Panjer points. We use these successive densities to improve the first approximation of the LDF. This step is illustrated in Figure 1.

3. To build this sequence of densities $\left(\tilde{g}^{\alpha, \beta}().\right)$, we consider the following kernel:

$$
K(h)=\left\{\begin{array}{l}
\frac{3}{4} \frac{\left(1-\frac{1}{5} h^{2}\right)}{\sqrt{5}}, \text { for }|h| \leq \sqrt{5} \\
0, \text { otherwise }
\end{array}\right.
$$

The bandwidth $h$ is calibrated with a cross-validation method based on the AMISE criterion (Wand and Jones (1995) and Silverman (1986)).

4. The Panjer recursion method is then applied on this new information set, and the density of the LDF is computed as follows:

$$
g(x)=p_{1} f(x)+\int_{0}^{x}\left(a+\frac{b y}{x}\right) f(y) g(x-y) d y, \text { where } x>0 .
$$

In expression (3.2), $p_{1}$ represents the frequency mass applied on the first Panjer point and $f(x)$ is the density of severities distributions.

- If $p_{1}$ is a Poisson distribution:

$$
g(x)=\lambda e^{-\lambda} f(x)+\frac{\lambda}{x} \int_{0}^{x} y f(y) g(x-y) d y, x>0,
$$

- If $p_{1}$ is a Binomial distribution:

$$
g(x)=\frac{p}{(1-p)}\left[N(1-p)^{N} f(x)+\int_{0}^{x}\left(\frac{(N+1) y}{(x-1)}\right) f(y) g(x-y) d y\right], x>0 .
$$

5. We approximate the integral in (3.2) using the trapezoidal rule [Rahman and Schmeisser (1990)]. Being in any point $x_{n}$, we get:

- If $p_{1}$ is a Poisson distribution:

$$
\tilde{g}\left(x_{n}\right)=\lambda e^{-\lambda} f\left(x_{n}\right)+\frac{\lambda}{x_{n}} \sum_{\alpha=1}^{n-1} \sum_{\beta=0}^{M} \frac{x_{\alpha, \beta} f\left(x_{\alpha, \beta}\right) \tilde{g}\left(x_{n}-x_{\alpha, \beta}\right)+x_{\alpha, \beta+1} f\left(x_{\alpha, \beta+1}\right) \tilde{g}\left(x_{n}-x_{\alpha, \beta+1}\right)}{2}\left(x_{n}-x_{\alpha, \beta}\right)
$$


- If $p_{1}$ is a Binomial distribution:

$$
\begin{aligned}
\tilde{g}\left(x_{n}\right) & =\frac{p}{1-p}\left[N(1-p)^{N} f(x)+\right. \\
& \left.\sum_{\alpha=1}^{n-1} \sum_{\beta=0}^{M} \frac{x_{\alpha, \beta} f\left(x_{\alpha, \beta}\right) \tilde{g}\left(x_{n}-x_{\alpha, \beta}\right)+x_{\alpha, \beta+1} f\left(x_{\alpha, \beta+1}\right) \tilde{g}\left(x_{n}-x_{\alpha, \beta+1}\right)}{2}\left(x_{n}-x_{\alpha,(\beta)}\right)\right]
\end{aligned}
$$

In these formulas $n$ is the number of Panjer points, $n \in[1, N] . M=2^{\zeta}, \zeta \in \mathrm{N}$, is the number of points between two Panjer points. The first point in the relationship (3.4) is denoted $x_{1,0}$ and is called a Panjer point. During the integration process, we use successively the Panjer points $x_{0}, x_{1}, x_{2} \ldots$ and the kernel points $\tilde{x}_{1 \beta}, \tilde{x}_{2 \beta}, \tilde{x}_{3 \beta} \ldots$ obtained in the previous step. The integration procedure works in the following way.

Algorithm 1. - Step 0 (Initialization): from $x_{1,0}$, we construct $\tilde{x}_{11}, \ldots, \tilde{x}_{1 M}$ and we use the full sequence $x_{0}, \tilde{x}_{11}, \ldots, \tilde{x}_{1 M}$ to compute $x_{2,0}$.

- Step 1: We define the set $A_{1}=\left(g\left(x_{0}\right), g\left(x_{1}\right), \tilde{g}_{21}, \ldots, \tilde{g}_{2 M}\right)$ on which we compute $g\left(x_{2}\right)$ using the relationship (3.4).

- Steps 2 to n: At each step, we define a new information set composed by the kernel and the Panjer recursion lattice $A_{k}, k=2, \ldots, n$ on which we calculate the values $\left(g\left(x_{0}\right), g\left(x_{1}\right), \ldots, g\left(x_{n}\right)\right)$.

6. The density associated to $g\left(x_{n}\right)$ provides the LDF, on which we are able to estimate a VaR.

\subsection{Numerical integration error}

Thanks to the previous algorithm, we built an approximation of the LDF. We now specify the errors made at each step. In the Panjer recursive formula (3.2), the term $\Phi(x)=p_{1} f(x)$ can be computed exactly, therefore the error is generated by the numerical integration of $\Psi(x, y)=$ $\int_{0}^{x} y f(y) g(x-y) d y$. Thus, in the following, we only present the error computed in this last term (see Appendix for details).

1. In the first step we use a Riemann sum to compute (3.2):

$$
g_{i}=\sum_{j=1}^{i}\left(a+\frac{b j}{i}\right) f_{j} g_{i-j} .
$$


Then, we evaluate the difference between (3.2) and (3.5) and we denote it :

$$
E 1=\left|p_{1} f(x)+\int_{0}^{x}\left(a+\frac{b y}{x}\right) f(y) g(x-y) d y-\sum_{j=1}^{i}\left(a+\frac{b j}{i}\right) f_{j} g_{i-j}\right| .
$$

This error tends to 0 as $i$ tends to infinity, nevertheless we consider that this error can be too large and cannot be used for risk management purpose.

2. In order to make the previous error smallest we consider the trapezoidal rule and we get the error E2. Let be $\Psi(x, y)=\int_{0}^{x} y f(y) g(x-y) d y$, then :

$$
E 2=\left|\Psi(x, y)-\sum_{\alpha=1}^{n-1} \frac{x_{\alpha} f\left(x_{\alpha}\right) \tilde{g}\left(x_{n}-x_{\alpha}\right)+x_{\alpha+1} f\left(x_{\alpha+1}\right) \tilde{g}\left(x_{n}-x_{\alpha+1}\right)}{2}\left(x_{n}-x_{\alpha}\right)\right|
$$

This error can be bounded as follows:

$$
E 2 \leq\left(\frac{1}{12 n}\right) \cdot \max _{y \in[0, n]}\left|\theta^{\prime \prime}(x, y)\right|
$$

where,

$$
\theta(x, y)=y f(y) g(x-y),
$$

and the second derivative $\theta^{\prime \prime}($.$) is computed with respect to y. Using the Leibnitz formula$ (see the Appendix for details), we can show that $\theta^{\prime \prime}($.$) is finite. Thus, the term E2 tends$ to zero as $n$ (the sample size) tends to $\infty$. We observe that the use of the trapezoidal rule enables us to reduce drastically the error E1. Nevertheless, this procedure is not sufficient to get consistent results for the VaR measure because of the lack of precision during the initialization time which is endogenous to the Panjer algorithm. We can remark that asymptotically the error $E 2$ tends to zero.

3. In practice we work with a finite sample, thus it is important to get a smaller error in that case. In order to solve this problem, we increase the initial information set on which we apply the Panjer algorithm, and the application of the trapezoidal rule provides the error E3: :

$E 3=\left|\Psi(x, y)-\sum_{\alpha=1}^{n-1} \sum_{\beta=0}^{M} \frac{x_{\alpha, \beta} f\left(x_{\alpha, \beta}\right) \tilde{g}\left(x_{n}-x_{\alpha, \beta}\right)+x_{\alpha, \beta+1} f\left(x_{\alpha, \beta+1}\right) \tilde{g}\left(x_{n}-x_{\alpha, \beta+1}\right)}{2}\left(x_{n}-x_{\alpha, \beta}\right)\right|$

and :

$$
|E 3| \leq\left(\frac{n}{12 M^{2}}\right) \cdot \max _{y \in[0, n]}\left|\theta^{\prime \prime}(x, y)\right|
$$


with $M>>n$, then $E 2>>E 3$. We can remark that the rate of convergence in (3.11) is in $M^{-1}$. As $M>>n$, this last error goes to zero very quickly, and depends on the lattice used in the algorithm. Thus, thanks to the new lattice we have introduced in the algorithm, we make the computation of the LDF more robust, improving its approximation.

\section{Results}

In order to protect the institution from a loss which could imply it to go bankrupt, it has been proposed to proceed in a VaR computation in order to decide the amount of capital allowed for this kind of risks. The simple method described in the previous section allows us to reach our goal in a faster and more accurate way than traditional algorithms.

We use our method to compute the VaR associated to this LDF. The results we present are based on simulation experiments, using parameters which have been estimated on real data sets. We recall that the regulator demands a security threshold of $99.9 \%(\alpha=0.1 \%)$ and we use it here.

Most results presented in this paper, such as the box-plots, are based on a convolution of a $\operatorname{Poisson}(\lambda)$ distribution with a $\operatorname{Lognormal}(\mu, \sigma)$ distribution. Furthermore, a short study of the sensitivity of the algorithm for different values of the distribution parameters is also provided.

The graphs are constructed using the following values: $M=2048$ and $h=15^{\frac{1}{5}}$ and the following parameters for the two distributions:

- Figure 2: $(\mu=0, \sigma=1)$, and $\lambda=25$

- Figure $3:(\mu=0.1, \sigma=1.2)$, and $\lambda=500$

- Figure 4: $(\mu=5, \sigma=2)$, and $\lambda=25$

We use the Monte Carlo simulations method $\left(\Omega=10^{6}\right)$ as a benchmark to compare the values got for the VaR by the different methods, and our algorithm uses successively 1.000, 10.000, 100.000 and 1.000.000 Poisson realisations. We change the generator's seed 100 times to create Box Plots. They provide the dispersion and the standard deviation of the VaR. In fine, four methods were implemented to compute it: 
1. Monte Carlo simulations (MC). We simulate the LDF using (1.1).

2. Kernel method (K). We simulate the LDF using (1.1) smoothed with an Epanechnikov kernel.

3. The LDF is simulated using the new algorithm discribed in Section $4.1(\mathrm{H})$.

4. The LDF is simulated using the Panjer's method (P) through relationship (3.2) .

The Figure 2 provides the results obtained for the first set of parameters. The bold line represents the value of the VaR obtained by each method. The results are given for the four samples. The box plots illustrate the dispersion around each VaR. In Figure 2, for several value of $N$, we observe that the three methods (a), (b) and (c) are competitive: the values of the VaR are very close. In that case our algorithm reduces drastically the dispersion around the VaR: thus our method reduces the error associated to the computation of the VaR. On the other hand the computation time is reduced comparing with traditional Monte Carlo methods. The Figure 3 provides the computation of the $\mathrm{VaR}$ for the set of parameters $(\mu=0.1, \sigma=1.2)$. The bias and the variance are always smaller when we use our method. The Figure 4 provides the results for the set of parameters $(\mu=5, \sigma=2)$, and $\lambda=25$. The Panjer algorithm, and our new algorithm are both competitive. Nevertheless, our algorithm provide always better results than the results obtained with the three other methods.

In Table 1, we compute the VaR using our hybrid method and the Luo and Shevchenko (2009) method, using a convolution of a Poisson $(\lambda)$ distribution and a $\operatorname{Lognormal}(0,2)$ distribution. We observe that our method performs better for $\lambda \geq 1$, and that it does not seem appropriate for very low $\lambda$. Indeed, when $0<\lambda<1$, the events are rare and our approach cannot capture correctly this phenomenon. It will be preferable to use a method based on extreme distributions. This will be the purpose of a companon paper.

We have also considered another set of distributions to compute the LDF and we provide the VaR for this set of distributions, comparing our method with the Monte Carlo simulations method. We observe that whatever the set of distributions we use, the results are close to the previous 
ones given in the figures 2,3 and 4 . The results are provided in Table $2^{2}$.

With almost any sets of parameters, the algorithm enable us to reduce, the bias and the variance of the estimated VaR measure.

\section{Conclusion}

In this paper, we have developed an innovative method to compute the LDF in order to provide the VaR associated to operational risks with a very small variance. The trade-off between variance reduction, computation speed and robustness is solved by our simple hybrid algorithm. The simulation time decreases by nearly $60 \%$ (in average) compared to Monte Carlo simulation. This point is fundamental for practitioners. At the same time the accuracy of our VaR results is very good compared to a VaR computed with a Monte Carlo simulation of a million of iterations.

Furthermore, the numerical integration error and the aliasing error due to the use of the Panjer recursion is reduced through the kernel interpolation. The error of approximation tends to zero as the number of interpolations points grows, and we have no initialisation bias for the LDF approximation. We have also shown that the choice of the distribution is not crucial in the method and it would theoretically work with GB2, Gumbel or g-and-h distributions. A carefull study of the impact of the number of intermediary points would be interesting, especially to deal with very low $\lambda$. Furthermore, to increase the computation speed, it might be interesting to assess the LDF initial support and consequently bypass the Monte Carlo simulation step.

Finally, our method computes the LDF and not only a quantile, which allows the aggregation of the 56 distributions implied by Basel II, taking into account dependencies - with copula, for example.

\footnotetext{
${ }^{2}$ The provided results were obtained with a computer with common capacities i.e. Pentium $4,3 \mathrm{GHz}$ and $1 \mathrm{~GB}$ of RAM. And we implemented the method with "R-2.10.0".
} 


\section{Appendix}

\subsection{The Panjer algorithm: a brief description}

Panjer (1981) provides a recursive formulation to compute high order convolutions. The main result is the following. Assuming that there exists two constants $a$ and $b$ such that

$$
p_{n}=p_{n-1}\left(a+\frac{b}{n}\right), \text { where } n=1,2,3 \ldots,
$$

then the following recursion holds for a regular function $g($.$) :$

$$
g(x)=p_{1} f(x)+\int_{0}^{x}\left(a+\frac{b y}{x}\right) f(y) g(x-y) d y, \text { where } x>0 .
$$

We can apply this recursion for the computation of the LDF. We consider the family of claim number distributions satisfying the recursion (6.1). In our case the Panjer class distribution either a Poisson distribution or a Binomial distribution:

- Poisson distribution, $p_{k}=\frac{e^{-\lambda} \lambda^{k}}{k !}$, where $\lambda \in \mathrm{R}^{+}$and $k \in \mathrm{N}$. $\frac{p_{k}}{p_{k-1}}=\left(\frac{\lambda}{k}\right)$, where $p_{0}=$ $e^{-\lambda}, a=0 b=\lambda$

- Binomial distribution, $p_{k}=\left(\begin{array}{c}n \\ k\end{array}\right) p^{x}(1-p)^{(n-k)}$, where $p \in[0,1]$ and $k \in \mathrm{N}$. $\frac{p_{k}}{p_{k-1}}=$ $\frac{(n-k+1) p}{(n(1-p))}$, where $p_{0}=(1-p)^{n}, a=-\frac{p}{(1-p)} b=\frac{n+1}{(1-p)}$

Then, using the formula (6.2), we get :

$$
g(x)=\left\{\begin{array}{l}
\sum_{n=1}^{\infty}=p_{n} f^{* n}(x), x>0 \\
0, \text { otherwise }
\end{array}\right.
$$

and $f(x)$ is any continuous type distribution for $\mathrm{x}>0$. Therefore, in our particular case, we could write the distribution as:

$$
g(x)=\lambda e^{-\lambda} f(x)+\frac{\lambda}{x} \int_{0}^{x} y / x f(y) g(x-y) d y .
$$

or,

$$
g(x)=\frac{p}{(1-p)}\left[N(1-p)^{N} f(x)+\int_{0}^{x}\left(\frac{(N+1) y}{(x-1)}\right) f(y) g(x-y) d y\right] x>0,
$$

In the case of a discret claim amount distribution, we use:

$$
g(x)=\frac{\lambda}{i} \sum_{j=1}^{i} j f_{i} g_{i-j} .
$$

or,

$$
g(x)=\frac{p}{(1-p)} \sum_{j=1}^{i} \frac{(n+1) j}{(i-1)} f_{i} g_{i-j}
$$




\subsection{Numerical Integration Error}

Right after the Monte Carlo simulation, using $\hat{G}$ got in step 1 of Subsection 2.1, we approximate this distribution and its density using Panjer algorithm.

We recall that any integrated function $\theta($.$) on a closed interval, such as \int_{0}^{x} \theta(y) d y$, can be approximated by :

$$
\sum_{j=0}^{N-1} \delta \sum_{i=1}^{s} \omega_{i} \theta\left(y_{j}+\alpha_{i} \delta\right),
$$

where $\delta=\frac{x}{N}, \omega \in R$ and, $N$ is the number of trapezes. Then, we rewrite the numerical integration error $E_{2}$ as :

$$
\left|\int_{0}^{x} y f(y) g(x-y) d y-\sum_{j=0}^{N-1} \delta \sum_{i=1}^{s} \omega_{i} \theta\left(y_{j}+\alpha_{i} \delta\right)\right| .
$$

The following theorem provides the expression of the previous approximation (6.9) as a function of the Peano Kernel, [Schatzman (1991)].

Theorem 6.1. Considering a p-order quadrature formula and a $k$ integer satisfying $k \leq p$. If $\theta:\left[y_{0}, y_{0}+\delta\right] \rightarrow \Re$ and $k$ continously differentiable times, the error is,

$$
E\left(\theta, y_{0}, \delta\right)=h^{k+1} \int_{0}^{1} N_{k}(\tau) \theta^{(k)}\left(y_{0}+\tau \delta\right) d \tau,
$$

where $\theta^{(k)}$ represents the $k^{\text {th }}$ derivative of $\theta$ and $N_{k}(\tau)$, the Peano kernel, is given by,

$$
N_{k}(\tau)=\frac{(1-\tau)^{k}}{k !}-\sum_{i=1}^{s} \omega_{i} \frac{\left(\alpha_{i}-\tau\right)_{+}^{k-1}}{(k-1) !} .
$$

The next proposition recall the main Peano Kernel properties: we are particularly interested by the fourth one.

Proposition 6.1. (Peano's kernel properties) Considering a p-order quadrature formula and a number $k$ satisfaying $1 \leq k \leq p$. We have :

1. $N_{k}^{\prime}(\tau)=-N_{k-1}(\tau)$ for $k \geq 2\left(\right.$ for $\tau \neq \alpha_{i}$ if $\left.k=2\right)$;

2. $N_{k}(1)=0$ for $k \geq 1$ if $\alpha_{i} \leq 1(i=1, \ldots, s)$;

3. $N_{k}(0)$ for $k \geq 2$ if $\alpha_{i} \geq 0(i=1, \ldots, s)$; 
4. $\int_{0}^{1} N_{p}(\tau) d \tau=\frac{1}{p !}\left(\frac{1}{p+1}-\sum_{i=1}^{s} \omega_{i} \alpha_{i}^{p}\right)=C$ (error constant $)$

5. $N_{1}(\tau)$ is fragmently linear, of slope coefficient -1 with jumps of height denoted $\omega_{i}$ at each points $\alpha_{i}(i=1, \ldots, s)$.

Now, we give a bound for the error (6.9), where the function $\theta$ is defined on a given interval :

Theorem 6.2. Let $\theta:[a, b] \rightarrow \Re k$ continously differentiable and a p-order quadrature formula $(p \geq k)$, then we have the following result

$$
|\tilde{E}| \leq h^{k} \cdot(b-a) \cdot \int_{0}^{1}\left|N_{k}(\tau)\right| d \tau \cdot \max _{x \in[a, b]}\left|\theta^{(k)}(x)\right|,
$$

where $h=\max _{j} h_{j}$.

From this theorem, we get the specific bound $E_{2}$ applying the previous result to (6.9):

$$
|\tilde{E}| \leq\left(\frac{1}{M}\right)^{2} \cdot(N) \cdot \frac{1}{12} \cdot \max _{y \in[0, N]}\left|\theta^{\prime \prime}(x, y)\right| .
$$

The derivative is computed with respect to y and is obtained using the Leibnitz formula which provides the $k^{\text {th }}$ derivatives of $\theta=u v$ when $u$ and $v$ are $C^{\infty}$ :

$$
\theta^{(k)}=\sum_{n=0}^{k}(k, n) u^{(n)} v^{(k-n)} .
$$

Then, for $u(y)=y f(y)$ and $v(y)=g(x-y)=g(x, y)$, we obtain :

$$
\frac{\partial^{2} \theta}{\partial y^{2}}=(2,0) y f(y) \frac{\partial^{2} g(x, y)}{\partial y^{2}}+(2,1)\left(f(y)+y \frac{\partial f(y)}{\partial y}\right) \frac{\partial g(x, y)}{\partial y}+(2,2)\left(2 \frac{\partial f(y)}{\partial y}+y \frac{\partial^{2} f(y)}{\partial y^{2}}\right) g(x, y) .
$$

And,

$$
\frac{\partial^{2} \theta}{\partial y^{2}}<\infty
$$




\section{References}

Artzner, P., Delbaen, F., Eber, J.-M. and Heath, D. (1999), 'Coherent measures of risk', Math. Finance 9 3, 203-228.

Bühlmann, H. (1984), 'Numerical evaluation of the compound poisson distribution: recursion or fast fourier transform?', Scan. Actuarial J. pp. 116-126.

Buch-Kroman, T. (2009), 'Comparison of tail performance of the champernowne transformed kernel density estimator, the generalized pareto distribution and the g-and-h distribution', Journal of Operational Risk 4.

Chernobai, A., Rachev, S. T. and Fabozzi, F. J. (2007), Operational Risk: A Guide to Basel II Capital Requirements, Models, and Analysis, John Wiley \& Sons.

Cooley, J. W. and Tukey, J. W. (1965), 'An algorithm for the machine calculation of complex fourier series', Math. Comput. 19, 297-301.

Craddock, M., Heath, D. and Platen, E. (2000), 'Numerical inversion of laplace transforms: a survey of techniques with applications to derivative pricing.', Computational Finance 4, 57-81.

Den Iseger, P. (2006), 'Numerical laplace inversion using gaussian quadrature.', Probability in the Engineering and Informational Sciences 20, 1-44.

Embrechts, P. and Frei, M. (2009), 'Panjer recursion versus fft for compound distributions.', Mathematical Methods of Operations Research .

Embrechts, P., Klüppelberg, C. and Mikosch, T. (2007), Modelling Extremal Events for Insurance and Finance, Springer, Berlin.

Fishman, G. S. (1996), Monte Carlo, Springer-Verlag, Berlin.

Frachot, A., Georges, P. and Roncalli, T. (2001), 'Loss distribution approach for operational risk', Working Paper, GRO, Crédit Lyonnais, Paris .

Grubel, R. and Hermesmeier, R. (1999), 'Computation of compound distributions 1: aliasing errors and exponential tilting.', ASTIN Bulletin 29, 197-214. 
Heckman, P. E. and Meyers, G. G. (1983), 'The calculation of aggregate loss distributions from claim severity and claim count distributions', Proceedings of the Casualty Actuarial Society 70, 22-61.

Hoaglin, D. C. (1985), 'Summarizing shape numerically: the g-and-h distributions', John Wiley ES Sons pp. 461-513.

Luo, X. and Shevchenko, P. (2009), 'Computing tails of compound distribution using direct numerical integration', Journal of Computational Finance .

Morgan, J. (1993), 'Riskmetrics'.

Panjer, H. (2006), Operational Risk: Modeling Analytics, Wiley, London.

Panjer, H. H. (1981), 'Recursive evaluation of a familly of compound distributions', Astin Bulletin 12, 22-26.

Peters, G. W., Johansen, A. M. and Doucet, A. (2007), 'Simulation of the annual loss distribution in operational risk via panjer recursions and volterra integral equations for value at risk and expected shortfall estimation.', Working Paper 2.

Pickands, J. (1975), 'Statistical inference using extreme oreder statistics', Annals of Statistics 3, 119-131.

Rahman, Q. I. and Schmeisser, G. (1990), Analytic Theory of Polynomials, Oxford Science Publications, New York.

Schaller, P. and Temnov, G. (2008), 'Efficient and precise computation of convolutions: applying fft to heavy tailed distributions.', Journal of Computational Methods in Applied Mathematics.

Schatzman, M. (1991), Analyse numérique, Masson, Paris.

Silverman, B. W. (1986), Density Estimation for statistics and data analysis, Chapman and Hall/CRC, London.

Wand, M. P. and Jones, M. C. (1995), Kernel Smoothing, Chapman and Hall/CRC, London. 


\begin{tabular}{|c|c|c|c|}
\hline$\lambda$ & Monte Carlo & Hybrid Panjer MC(new) & Direct Numerical Integration \\
\hline 0.1 & 106.57 & 82.67 & 105.38 \\
\hline 1 & 483.03 & 487.327 & 490.55 \\
\hline 10 & 1749.564 & 1782.896 & 1779.2 \\
\hline $10^{2}$ & 5857.304 & 5849.909 & 5861.7 \\
\hline $10^{3}$ & 21323.38 & 21343.86 & 21149 \\
\hline $10^{4}$ & 107964.6 & 108557.8 & 108350 \\
\hline
\end{tabular}

Table 1: Sensitivity analysis and algorithm comparison 


\begin{tabular}{|c|c|}
\hline Smoothed Monte Carlo Simulations $\left(10^{6}\right.$ iterations) & Hybrid Panjer-MC (new) \\
\hline Poisson(30)-Weibull $(2,3)$ & \\
\hline 136.127 & 136.217 \\
\hline Poisson(10)-Weibull $(1,1)$ & 27.996 \\
\hline 28.010 & 45.969 \\
\hline Poisson(10)-GPD(1,1,0) & \\
\hline 46.302 & 161.912 \\
\hline Poisson(20)-GPD(1,3,0) & 32.931 \\
\hline 161.889 & \\
\hline Binomial(0.2,10)-logNormal $(0,1)$ & 1997.123 \\
\hline 31.038 & \\
\hline Binomial(0.4,5)-logNormal $(1,2)$ & 601999.3 \\
\hline 1988.161 & \\
\hline Poisson(50)-logNormal $(5,2)$ & 2901.745 \\
\hline 601999.3 & \\
\hline Poisson(25)-logNormal $(0,2)$ & \\
\hline 2924.167 & \\
\hline
\end{tabular}

Table 2: VaR results 


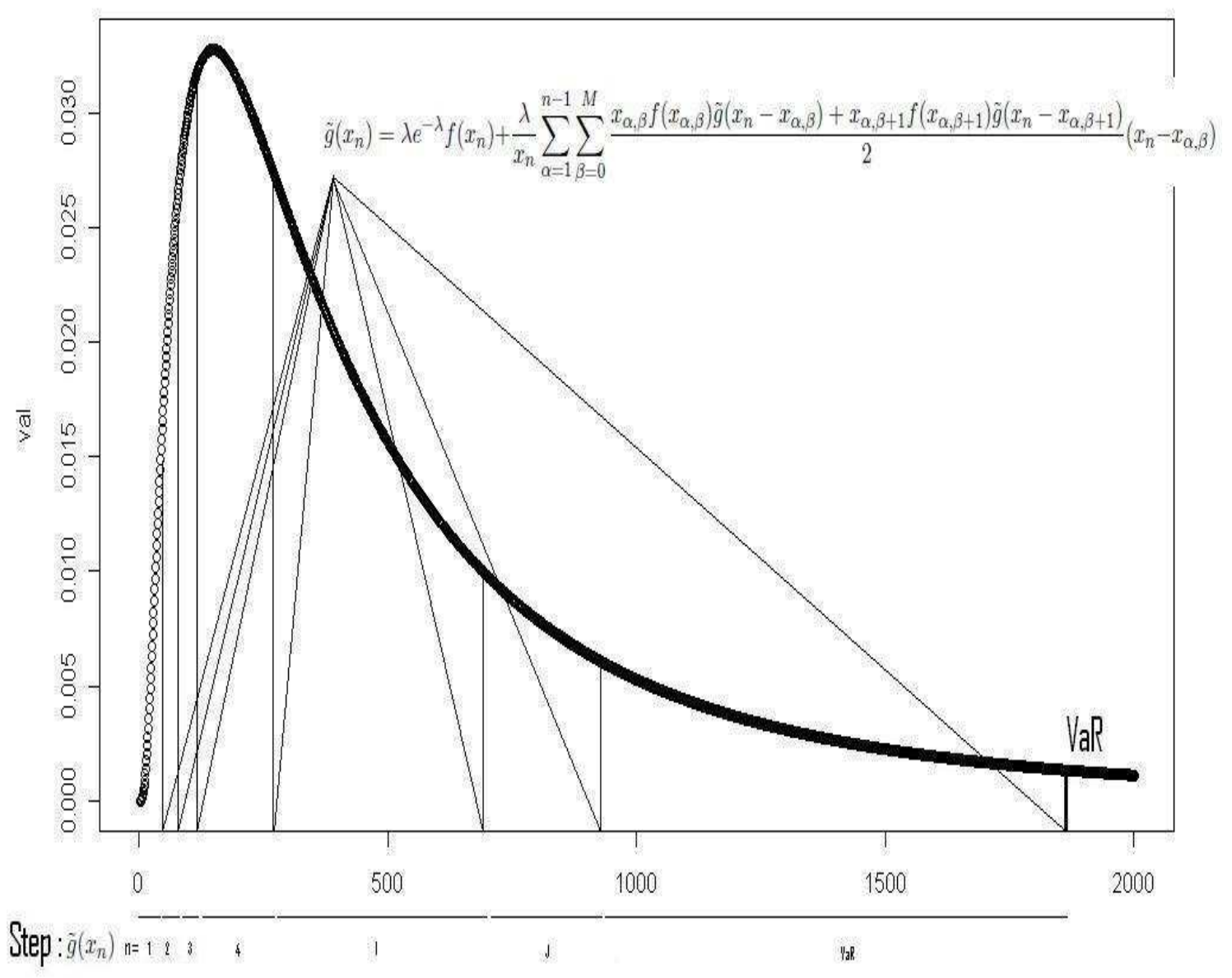

Figure 1: Details of the steps 2-3-4 to approximate the LDF 

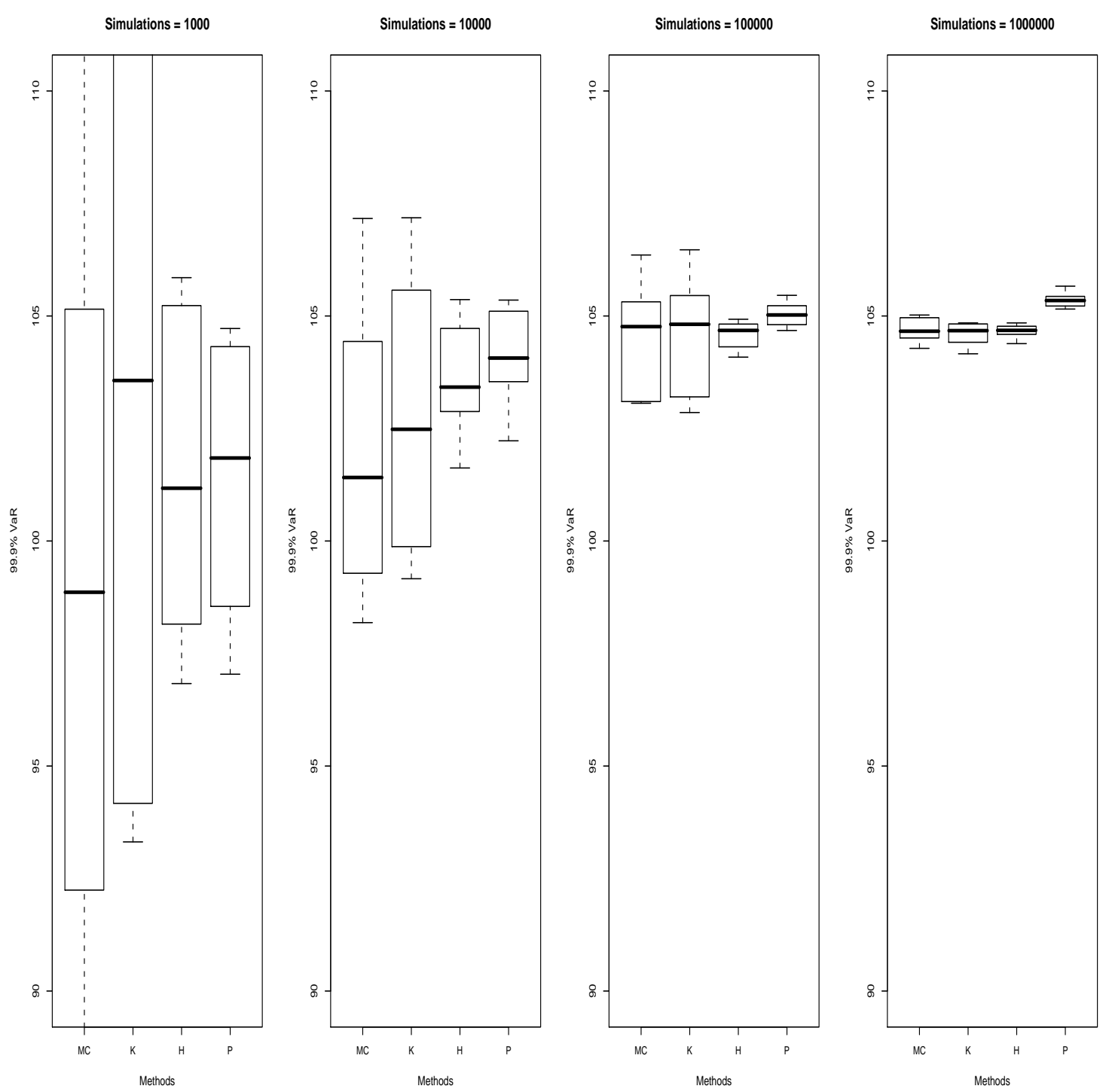

Figure 2: Box plots obtained from the four methods: $(\mu=0, \sigma=1)$, and $\lambda=25$ 

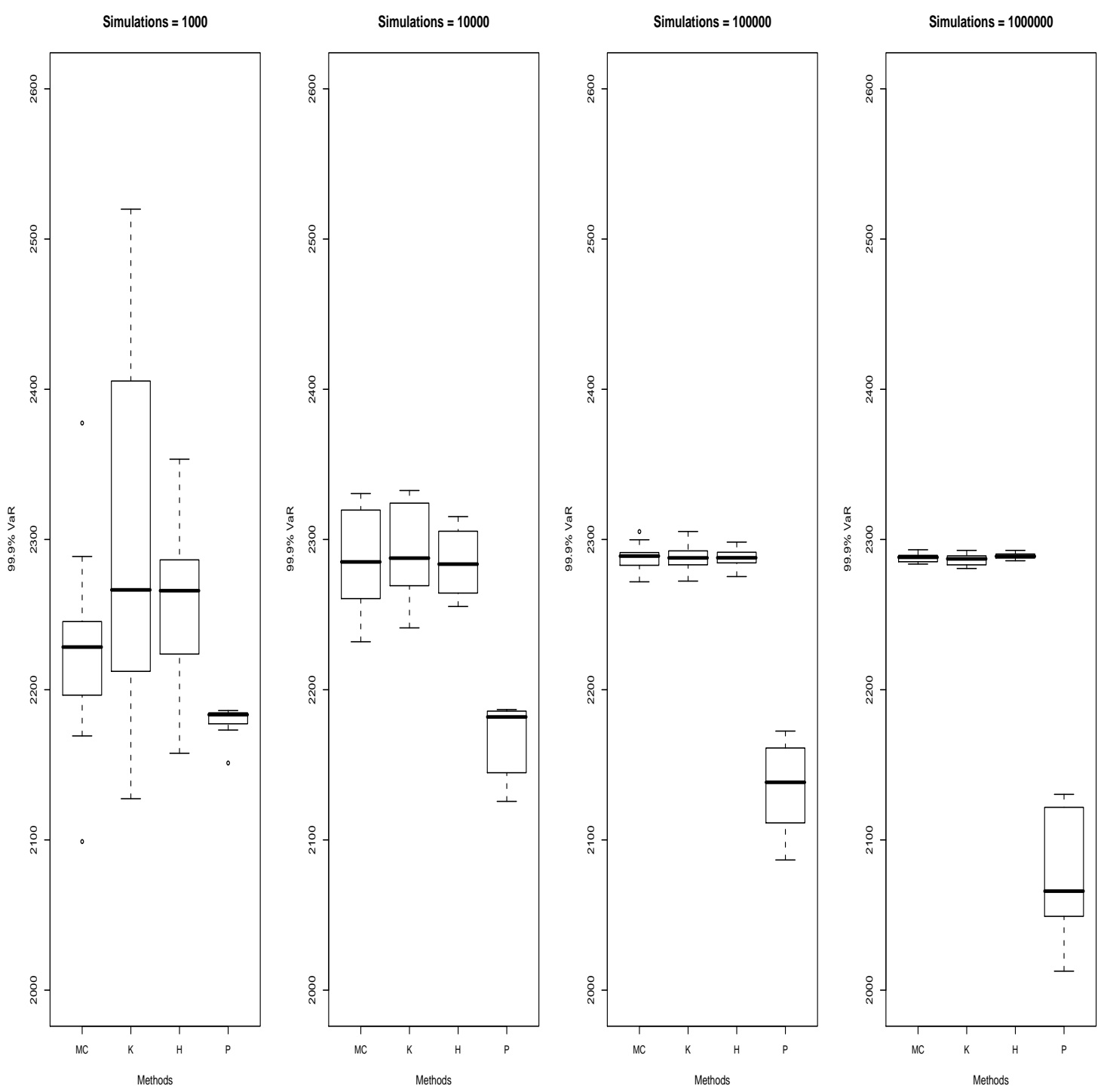

Figure 3: Box plots obtained from the four methods: $(\mu=0.1, \sigma=1.2)$, and $\lambda=500$ 

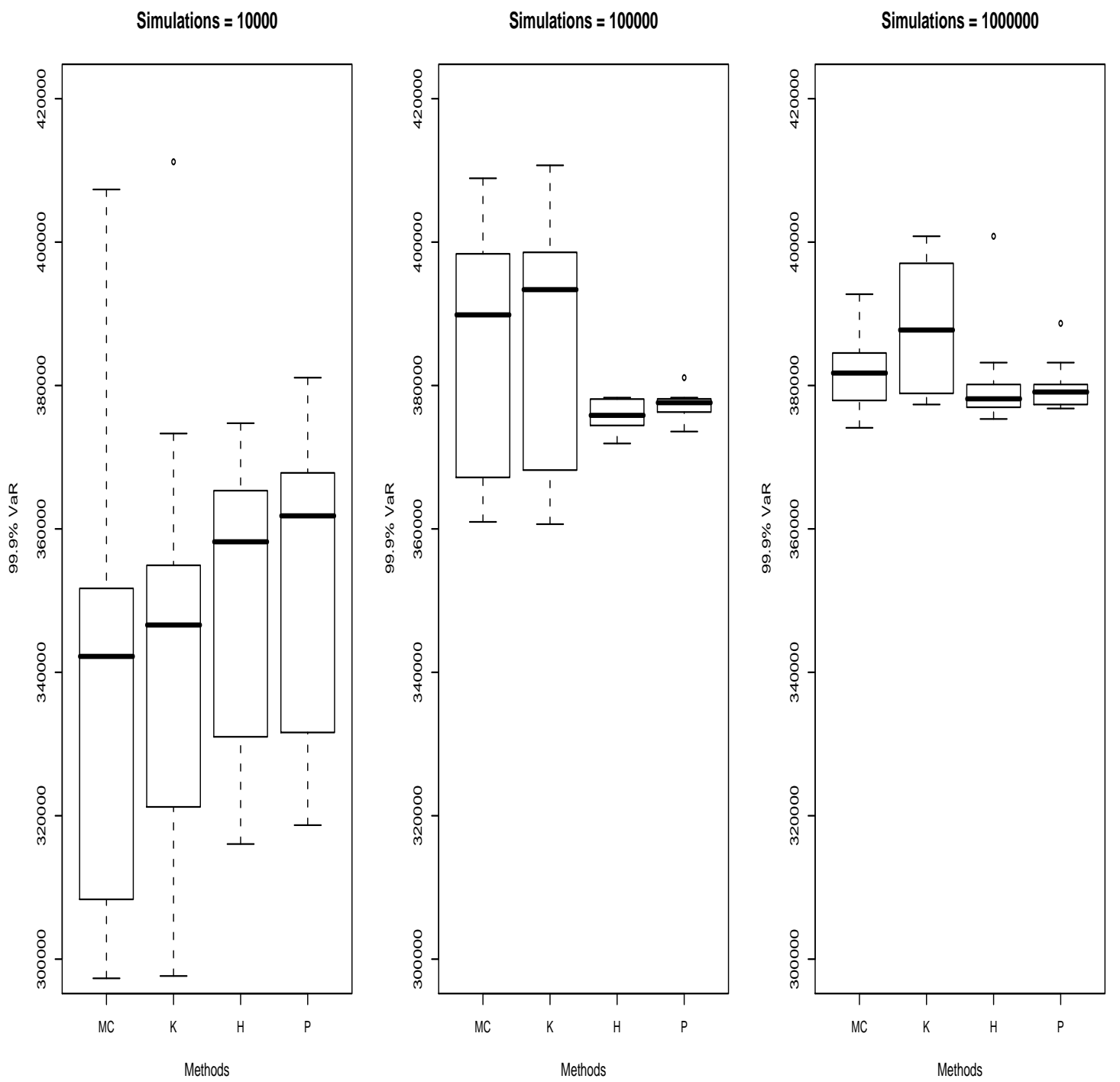

Figure 4: Box plots obtained from the four methods: $(\mu=5, \sigma=2)$, and $\lambda=25$ 